\title{
Photon echo from an ensemble of (In, Ga)As quantum dots
}

(C) la.A. Babenko ${ }^{1}$, I.A. Yugova ${ }^{1}$, S.V. Poltavtsev ${ }^{1,2}$, M. Salewski ${ }^{2}$, I.A. Akimov ${ }^{2,3}$, M. Kamp ${ }^{4}$, S. Höfling ${ }^{4}$, D.R. Yakovlev ${ }^{2,3}$, M. Bayer ${ }^{2,3}$

${ }^{1}$ Spin Optics Laboratory, St. Petersburg State University,

198504 St. Petersburg, Russia

${ }^{2}$ Experimentelle Physik 2, Technische Universität Dortmund,

44221 Dortmund, Germany

${ }^{3}$ loffe Institute,

194021 St. Petersburg, Russia

${ }^{4}$ Technische Physik, Universität Würzburg,

D-97074 Würzburg, Germany

E-mail: babenkojana@gmail.com

Photon echo from trions and excitons in (In,Ga)As/GaAs quantum dots was studied theoretically and experimentally. Theoretical analysis allowed us to distinguish between photon echo signals from excitons and trions measured in the same range of wavelength using different polarization configurations of laser excitation. The theoretical predictions are in good agreement with the experimental data.

\section{Acknowledgement}

The authors thank Doctor Christian Schneider for assisting in the growth of the sample. This work has been supported in part by the Russian Science Foundation (Grant No 14-42-00015) and Deutsche Forschungsgemeinschaft in the framework of ICRC TRR 160. 